\title{
Prenatal maternal sleep
}

\begin{tabular}{|c|}
\hline $\begin{array}{c}\text { Diana-Antonia Iordachescu}{ }^{1} \text {, Corina-Ioana Paica }{ }^{1} \text {, Elena Otilia Vladislav }{ }^{1} \text {, } \\
\text { Ana-Ilinca Ilie }{ }^{1} \text {, Corina Gica }{ }^{2,3} \text {, Gheorghe Peltecu }{ }^{2,3} \text {, Anca Maria Panaitescu }{ }^{2,3} \text {, } \\
\text { Nicolae Gica }{ }^{2,3}\end{array}$ \\
\hline $\begin{array}{l}{ }^{1} \text { Faculty of Psychology and Educational Sciences, University of Bucharest, Romania } \\
\text { 2"Carol Davila" University of Medicine and Pharmacy, Bucharest, Romania } \\
\qquad{ }^{3} \text { Filantropia Clinical Hospital, Bucharest, Romania }\end{array}$ \\
\hline
\end{tabular}

\begin{abstract}
Pregnancy affects women's sleep in many ways. Physical and emotional difficulties, especially towards the end of pregnancy, can lead to sleep disorders. Several studies suggest associations between sleep quality and high blood pressure, diabetes and depression. Sleep deprivation affect both mother and fetus. Sleep disorders are associated with low birth weight, intrauterine growth restriction, premature birth and cesarean births.

This paper is a review based on information from the literature. The analysis was limited to articles and guidelines in English published between January 1, 2000 and May 1, 2020 on PubMed, ScienceDirect and Google Scholar using the following keywords: sleep, pregnancy, depression, anxiety, mental health, sleep disorders, pregnant women, interventions, treatment. In this review, we discuss the characteristics of prenatal maternal sleep, hormonal changes during pregnancy and their effects on sleep, the effects of changing sleep patterns on the pregnant woman, and the interventions needed to optimize sleep quality.

According to the literature, sleep disorders are significant risk factors for mood disorders. Knowing sleep changes and their effects is useful for informing mothers. Consideration of non-pharmacological treatments and interventions such as cognitive-behavioral therapy, mindfulness therapy and relaxation exercises can be effective in optimizing the quality of sleep in pregnant women.
\end{abstract}

Keywords: sleep, pregnancy, depression, anxiety, mental health, sleep disorders, pregnant women, interventions, treatment

\section{INTRODUCTION}

For most women, pregnancy is a special experience that is most often accompanied by substantial changes regarding both their physical and emotional well-being. The enthusiasm and joys of becoming a mother are commonly intertwined with concerns, questions, curiosities and decidophobia. Whilst some would describe pregnancy as the most beautiful experience a woman could have, others depict pregnancy as a demanding process that oftentimes includes hardships such as: back pain, apathy, muscle cramps, eating difficulties etc. Pregnancy is generally portrayed as a challenging experience that incorporates both physical changes and emotional trials, factors which can trigger the appearance of sleep disorders.

Restful sleep is pivotal for good health, as most studies suggest [1-3]. It is reciprocally linked to the: improvement of the functioning of the im- mune system, the suppression of cancer cell growth, the balance between one's metabolism and hormone levels and the regulation of blood glucose and blood pressure. It also helps fight off fatigue. Amongst the numerous beneficial aspects of restful sleep, improved problem-solving skills, memory and concentration, superior emotional self-regulation and coping mechanisms, as well as maximized creativity are noted $[4,5]$.

Pregnancy is a state commonly associated with sleep deprivation. The effects of lack of proper sleep may be more pronounced in pregnant women than in non-pregnant women. The body of a pregnant woman is constantly maintaining a balance that allows the fetus to obtain as many resources as possible, without neglecting or affecting the needs and nutritional reserves of the mother [6].

A multitude of factors play a role in the development of sleep deprivation and sleep disorders in pregnant women. Sleep deprivation carries numer- 
ous risks for both mother and child. This article aims to showcase recent studies focused on prenatal maternal sleep and the emotional, cognitive and behavioral changes involved.

\section{CHARACTERISTICS OF PRENATAL MATERNAL SLEEP}

On average, adults need seven to nine hours of sleep in a twenty-four hour period. A young, healthy adult spends about $5 \%$ of the night being awake, $20 \%$ in a deep sleep, which is associated with restfulness and $55 \%$ in a light sleep. Rapid eye movement (REM) is a phase of intense oculomotor activity, which customarily amounts to 20$25 \%$ of sleep and is considered to be an important predictor of proper cognitive functioning.

Sleep requirements vary between individuals and are doubtlessly influenced by age, gender and state of pregnancy. During pregnancy sleep patterns are altered, oftimes in a negative manner, the contributory factor routinely cited being the increased intensity of disruptive physical factors [7]. Pregnancy has a duration of roughly 40 weeks, which can be divided into three trimesters. Each trimester is unique in terms of fetal development and physiological changes occurring in the body of the pregnant woman. As such, there are notable differences in terms of quality and sleep requirements during the three trimesters of pregnancy.

In the first trimester (0-13 weeks), the duration of sleep tends to increase compared to pre-pregnancy. The sum total of hours of sleep per night increases by 0.7 hours in the first trimester, but sleep efficiency decreases compared to the period before pregnancy. It seems that longer sleep duration is needed in order to compensate for decreased sleep efficiency [8].

During the second trimester of pregnancy (1426 weeks), the duration of sleep becomes similar to the levels of sleep registered before pregnancy, thus helping reduce fatigue.

Throughout the third trimester of pregnancy (27-40 weeks), various disturbing factors occur that perturb the quality and duration of sleep (such as: snoring, restless legs syndrome, frequent urination, back pain, heartburn, increased fetal movement at nighttime or general discomfort). A study conducted by Facco et al. [9] demonstrated that sleep disorders are prevalent in primiparas women and significantly increase during pregnancy. Starting with the third trimester of pregnancy, women reported shorter sleep duration along with reduced sleep quality, mostly caused by breathing difficulties and snoring.
Lack of restful sleep can affect one's overall health and can lead to serious medical conditions. Sleep deprivation plays a pivotal role in the appearance of irritability, fatigue, apathy, lack of enthusiasm in carrying out social and professional activities and is also a predictor of the onset of prenatal depression [10].

\section{HORMONAL FACTORS AFFECTING SLEEP IN PREGNANCY}

Hormonal changes and general physical discomfort affect the quality of sleep, especially in pregnant women [11]. The first hormonal change that occurs in the body of a pregnant woman is the production of hCG (human chorionic gonadotropin), which stimulates the production of progesterone in the first 6 to 8 weeks of pregnancy [12]. The longer sleep duration observed in the first trimester of pregnancy might be linked to the increased levels of the hCG. Estrogen decreases REM sleep [13]. Progesterone, on the other hand, has a reported hypnotic, sedative or anesthetic effect, induces NREM sleep $[13,14]$ and decreases REM latency [15].

\section{THE EFFECTS OF CHANGING SLEEP PATTERNS IN PREGNANT WOMEN}

Pregnancy requires adaptation to an ever-changing body and unfamiliar sleep patterns. Establishing favorable sleep patterns is highly beneficial for both the physical and psychological well-being of a pregnant woman. Proper sleep is crucial for maintaining homeostasis and also helps counterbalance the negative effects of stress.

Sleep deficits can contribute to perceived pregnancy-related difficulties and thereby cause sleep disturbances, reduced sleep duration or insomnia. Sleep deprivation can also disrupt the functioning of brain areas involved in the interpretation and regulation of stress responses, such as the amygdala [16] and hippocampus [17]. This prompts an additional activation of the stress system. Consequently, sleep deprivation can be considered a psychological stressor in itself. Prenatal maternal sleep deficit can lead to a vicious cycle, in which the sleep deficit during pregnancy is considered both the result of stress and a stressor in itself [18].

Disturbed sleep causes adverse health consequences, including fatigue, decreased cognitive focus and altered mood, in both pregnant and non-pregnant women. Sleep disorders during pregnancy are considered to be significant risk factors 
for the onset of mood disorders and depression [19].

Postpartum depression is a relatively common mood disorder: about $10-15 \%$ of fertile women have postpartum depression [20]. Epidemiological studies suggest that there is a link between sleep disorders and depression [21]. Women with postpartum depression displayed a history of decreased sleep levels or sleep quality starting from late pregnancy/the third trimester up to the first few weeks after birth, whilst women who did not have symptoms of postpartum depression had a reported increase in total sleep time over the same time period [22].

Studies surmise that pregnant women routinely score lower on specific cognitive tasks compared to non-pregnant women [23]. Ziomkiewicz et al. [6] argue that elevated cortisol levels during pregnancy affect maternal cognitive function due to a long-term effect on hippocampal structure and function. They claim that maternal cognitive changes occur due to a compromise in energy availability for both the fetus and mother. Elevated cortisol levels during pregnancy increase energy flow from the mother to the fetus through the placenta, in order to support fetal development [24]. Hence, the energy available for the maternal brain is used to improve cognitive processes that are beneficial to the health of the mother and fetus, leaving less energy for secondary cognitive processes.

Most types of memory have a deficit in pregnant women compared to the control group, with the exception of recognition memory, which is reported to be superior during pregnancy. Better facial recognition fulfills the function of protection, as it makes women more socially attentive, aiding them in the identification of people and situations that will provide them with safety.

\section{METHODS OF TREATMENT}

Insufficient total sleep or fragmentation of deep sleep in a pregnant woman are leading causes in the appearance of developmental or growth problems in the unborn baby, with further potential risk factors for the pregnancy itself. It is crucial that specialists find ways to help pregnant women improve sleep quality. Due to concerns about medication use during pregnancy, many types of medication being contraindicated during this period [8], psychological interventions must be considered.

Cognitive behavioral therapy for insomnia is an effective non-pharmacological, skills-based meth- od of psychotherapy treatment for insomnia during pregnancy, as indicated by a study developed by Manber et al. [25]. They offered pregnant women cognitive-behavioral therapy sessions for insomnia (a psychosocial, empirically supported intervention that addressed sleep-related behaviors and cognitions) and the control group exercises (in which pregnant women were asked to associate images with disturbing experiences which occurred at night with neutral images identified by the patient). Women who underwent CBT sessions for insomnia were able to successfully downgrade the severity of their insomnia, with reported cases of full remission, as well as register a significant decrease in postnatal depression scores.

Mindfulness is based on Eastern contemplative traditions and it is "inherently a state of consciousness" that involves moment-by-moment awareness of thoughts, emotions, sensations, and the environment [26]. Mindfulness interventions demonstrate similar improvements in quality of sleep symptoms and insomnia in pregnant women [27]. Studies also show that with the optimization of sleep, the symptoms of anxiety and depression in pregnant women decrease [28].

Psychological interventions that include relaxation exercises [29] (listening to music, guided imagery relaxation intervention, muscle relaxation, and breathing techniques to reduce arousal and promote sleep) have been shown to be effective in optimizing sleep quality [30-32], sleep duration and also pregnant women stated there were fewer sleep interruptions during pregnancy [33].

Studies also show that longitudinal sleep education programs, designed to increase sleep knowledge and improve sleep practices, help pregnant women optimize their sleep quality, initiation and continuity [34].

When psychological interventions offer unsatisfactory results, pharmacotherapy may be considered. A thorough risk-benefit analysis is recommended to be performed in advance, since drug treatments carry the risk of teratogenicity or of other unfavorable secondary effects on the pregnant woman or the fetus.

Pregnancy frequently causes women to be more cautious and have more concerns about drug treatments. These concerns ought to be taken seriously, as there are still many unknown aspects regarding the exact mechanisms and pathways involved in the relationship between sleep disorders and pharmacotherapy. In addition, further research on the risks and secondary effects of pharmacotherapy during pregnancy is needed. 
Sleep issues are common during pregnancy. The most common complaints of women during pregnancy consist of sleep disturbance by failure to fall asleep quickly, restlessness during the night and waking up often during the night, challenges which continue to be present in the first year postpartum. There is also a reported significant change in sleep duration during the pregnancy, from an increase in the first trimester to a fairly large decrease towards the end of the pregnancy [35].

Physical and emotional challenges, especially towards the end of pregnancy, can lead to sleep disturbances. Hormonal changes during pregnancy may further affect sleep. Estrogen decreases REM sleep, while progesterone increases NREM sleep. Suppressing REM sleep could be beneficial since it stabilizes both maternal and fetal autonomic nerve functions, such as blood pressure, heart rate and respiratory rate. Sleep deprivation is considered both the result of stress and a stressor in itself. Several researchers have reported associations between poor prenatal maternal sleep quality and high blood pressure, diabetes and depression. Recent studies [9] have shown correlations between sleep disorders and their adverse effects on an individual's health. However, these studies did not include pregnant women, containing little evidence of an association between poor sleep quality during pregnancy and its negative effects on maternal and fetal health.

The hypothesis that sleep disorders correlate with complications during pregnancy is biologically plausible. First of all, pregnancy is in itself associated with sleep disturbance. Insomnia, snoring and restless legs syndrome are frequently reported by pregnant women. Secondly, the health issues generated by sleep disorders, such as hypertension, diabetes and depression have correspondents during pregnancy: gestational diabetes, gestational hypertension and pre- and post-natal

Conflict of interest: none declared

Financial support: none declared

\section{REFERENCES}

1. Scott JPR, McNaughton LR, Polman RCJ. Effects of sleep deprivation and exercise on cognitive, motor performance and mood Physiol Behav. 2006;87:396-408

2. Guadagni V, Burles F, Ferrara M, laria G. The effects of sleep deprivation on emotional empathy. J Sleep Res. 2014;23:657-663.

3. Boonstra TW, Stins JF, Daffertshofer A, Beek PJ. Effects of sleep deprivation on neural functioning: an integrative review. Cell Mol Life Sci. 2007;64:934-946.

4. Lewis PA, Knoblich G, Poe G. How Memory Replay in Sleep Boosts Creative Problem-Solving. Trends Cogn Sci. 2018;22:491-503.

5. Vandekerckhove M, Cluydts R. The emotional brain and sleep: An intimate relationship. Sleep Med Rev. 2010;14:219-226.

6. Ziomkiewicz A, Wichary S, Jasienska G. Cognitive costs of reproduction: life-history trade-offs explain cognitive decline during depression. The first step in assessing the impact of sleep disorders and their potential contributions to obstetrical and neonatal outcomes is to fully understand the prevalence and patterns of prenatal maternal sleep [9]. Sleep disorders in pregnancy are often associated with low birth weight, intrauterine growth problems, preterm birth and deliveries by cesarean section [36].

\section{CONCLUSIONS}

Disruption or deprivation of sleep during pregnancy remains a significant risk factor for the appearance of mood disorders. The implications of untreated sleep deprivation on the health of the pregnant woman and the fetus must be taken into account when deciding whether treatment of sleep disorders or sleep deficiencies is necessary.

To improve the quality of sleep during pregnancy, treatment should be personalized. Non-pharmacological treatments and psychological interventions against sleep deprivation could be effective, but further research is needed in order to increase its efficiency.

In addition to researching the pathways, mechanisms, and treatments involved in sleep deprivation during pregnancy, it is important to consider how society influences the quality of sleep in pregnant women. Being knowledgeable about sleep changes and the possible outcomes of improper sleep during pregnancy may prove to be useful for mothers who expect pre-delivery checks. Further adjustment of medical policies must be made. All parties involved in monitoring the development of the pregnancy (such as pregnant women and doctors) must be informed about the possible obstetrical and neonatal outcomes, as well as the possible treatment methods of sleep deprivation before, during and after pregnancy.

pregnancy in women. Biol Rev Camb Philos Soc. 2019 94(3):1105-1115.

7. Sahota PK, Jain SS, Dhand R. Sleep disorders in pregnancy. Curr Opin Pulm Med. 2003;9(6):477-483.

8. Oyiengo D, Louis M, Hott B, Bourjeily G. Sleep disorders in pregnancy. Clin Chest Med. 2014;35(3):571-587.

9. Facco FL, Kramer J, Ho KH, Zee PC, Grobman WA. Sleep Disturbances in Pregnancy. Obstet Gynecol. 2010;65:220-222.

10. Skouteris H, Wertheim EH, Germano C, Paxton SJ, Milgrom J. Assessing sleep during pregnancy: a study across two time points examining the Pittsburgh Sleep Quality Index and associations with depressive symptoms. Womens Health Issues. 2009;19:45-51.

11. Lee KA, Zaffke ME, McEnany G. Parity and sleep patterns during and after pregnancy. Obstet Gynecol. 2000;95:14-18. 
12. Pierce J,GB, Midgley J,AR. The origin and function of human syncytiotrophoblastic giant cells. Am J Pathol. 1963;43:153-173.

13. Nishina H, Honda K, Okai T, Kozuma S, Inoué S, Taketani Y. Characteristic changes in sleep patterns during pregnancy in rats. Neurosci Lett. 1996;203:5-8.

14. Friess E, Tagaya H, Trachsel L, Holsboer F, Rupprecht R. Progesterone-induced changes in sleep in male subjects. Am J Physiol. 1997;272(5 Pt 1):E885-E891.

15. Lee KA, Shaver JF, Giblin EC, Woods NF. Sleep patterns related to menstrual cycle phase and premenstrual affective symptoms. Sleep (New York). 1990;13:403-409.

16. Drevets WC. Prefrontal Cortical-Amygdalar Metabolism in Major Depression. Ann N Y Acad Sci. 1999;877:614-637.

17. Duman RS. Depression: a case of neuronal life and death? Biol Psychiatry. 2004;56:140-145.

18. Palagini L, Gemignani A, Banti S, Manconi M, Mauri M, Riemann D. Chronic sleep loss during pregnancy as a determinant of stress: impact on pregnancy outcome. Sleep Med. 2014;15:853-859.

19. Swanson LM, Pickett SM, Flynn H, Armitage R. Relationships Among Depression, Anxiety, and Insomnia Symptoms in Perinatal Women Seeking Mental Health Treatment. J Womens Health (Larchmt). 2011;20:553-558.

20. Nielsen Forman D, Videbech P, Hedegaard M, Dalby J, Secher NJ. Postpartum depression: identification of women at risk. BJOG. 2000;107:1210-1217.

21. Breslau N, Roth T, Rosenthal L, Andreski P. Sleep disturbance and psychiatric disorders: A longitudinal epidemiological study of young Adults. Biol Psychiatry. 1996;39:411-418.

22. Wolfson AR, Crowley SJ, Anwer U, Bassett JL. Changes in Sleep Patterns and Depressive Symptoms in First-Time Mothers: Last Trimester to 1-Year Postpartum. Behav Sleep Med. 2003;1:54-67.

23. Anderson MV, Rutherford MD. Recognition of Novel Faces after Single Exposure is Enhanced during Pregnancy. Evol Psychol. 2011;9:47-60.

24. Gangestad SW, Caldwell Hooper AE, Eaton MA. On the function of placental corticotropin-releasing hormone: a role in maternal-fetal conflicts over blood glucose concentrations. Biol Rev Camb Philos Soc. 2012;87:856-873.
25. Manber R, Bei B, Simpson N, et al. Cognitive Behavioral Therapy for Prenatal Insomnia: A Randomized Controlled Trial. Obstet Gynecol. 2019;133:911-919.

26. Grabovac AD, Lau MA, Willett BR. Mechanisms of Mindfulness: A Buddhist Psychological Model. Mindfulness. 2011;2:154-166.

27. Beddoe AE, Lee KA, Weiss SJ, Powell Kennedy H, Yang CPP. Effects of mindful yoga on sleep in pregnant women: a pilot study. Biol Res Nurs. 2010;11:363e70.

28. Field T, Diego M, Delgado J, Medina L. Tai chi/yoga reduces prenatal depression, anxiety and sleep disturbances. Complement Ther Clin Pract. 2013;19:6e10.

29. Bacaro V, Benz F, Pappaccogli A, et al. Interventions for sleep problems during pregnancy: A systematic review. Sleep Med Rev. 2020;50:101234-101234.

30. Schaffer L, Jallo N, Howland L, James K, Glaser D, Arnell K. Guided imagery: an innovative approach to improving maternal sleep quality. J Perinat Neonatal Nurs. 2013;27:151e9.

31. Liu YH, Lee CS, Yu CH, Chen $\mathrm{CH}$. Effects of music listening on stress, anxiety, and sleep quality for sleep-disturbed pregnant women. Women Health. 2016;56:296e311

32. Ozkan SA, Rathfisch G. The effect of relaxation exercises on sleep quality in pregnant women in the third trimester: a randomized controlled trial. Complement Ther Clin Pract. 2018;32:79e84.

33. Lee KA, Gay CL, Alsten CR. Sleep enhancement training for pregnant women. Obstet Gynecol. 2016;128:964.

34. Ladyman C, Gander P, Huthwaite M, Sweeney B, Signal TL. Sleep HAPi: A Feasibility and Descriptive Analysis of an Early and Longitudinal Sleep Education Intervention for Pregnant Women. Behav Sleep Med. 2020:1-18.

35. Hedman C, Pohjasvaara T, Tolonen U, Suhonen-Malm AS, Myllylä VV. Effects of pregnancy on mothers' sleep. Sleep Med. 2002; 3:37-42.

36. Nodine PM, Matthews EE. Common Sleep Disorders: Management Strategies and Pregnancy Outcomes. J Midwifery Womens Health. 2013;58:368-377. 